\title{
4D-STEM Quantification of Nanoscale Ordered Domains in Organic Semiconducting Polymers
}

\author{
Gabriel Calderon Ortiz ${ }^{1}$, Menglin Zhu ${ }^{1}$, Letian Dou ${ }^{2}$ and Jinwoo Hwang ${ }^{1}$ \\ ${ }^{1}$ The Ohio State University, Columbus, Ohio, United States, ${ }^{2}$ Purdue University, West Lafayette, Indiana, \\ United States
}

Recent advances in organic photovoltaics (OPVs) have provided promising new routes to produce affordable and flexible solar cell devices. The main challenge so far is the relatively low efficiency of these devices as compared to their inorganic counterparts. To improve their photovoltaic efficiency, a thorough investigation of structure-property relationships at the nanoscale is required, because their important properties, including carrier mobility, mechanical and optical properties, can be controlled by the nanoscale ordering of the constituent molecules [1]. Scanning transmission electron microscopy (STEM) provides ideal probe sizes to resolve the required information at the nanoscale, although the effect of radiation damage must be reduced to avoid any complication in the data analysis.

In this work, we demonstrate a novel way to acquire spatially resolved information about the molecular ordering in OPV using 4-dimensional STEM (4D-STEM) based on the new generation fast pixelated STEM detector (Fig 1a and 1b). Our characterization combines high spatial resolution ( $1 \mathrm{~nm})$ and high dynamic range (32-bit) in signal detection, which allows for quantitative determination of the details of the ordering parameters, including the type, size, and volume fraction of the ordered domains with unprecedented precision. To minimize the effects of radiation damage and sample contamination, we use low dose conditions at low accelerating voltages $(60-80 \mathrm{keV})$, combined with short dwell times $(\sim 1 \mathrm{~ms})$. The OPV samples were annealed at temperatures between 100 and $120 \mathrm{C}$ in situ to eliminate carbon contamination of the sample by the beam exposure.

We studied Poly(3-hexylthiophene-2,5-diyl) (P3HT) and P3HT: Phenyl-C61-butyric acid methyl ester (PCBM) (P3HT: PCBM). These polymers have been used as one of the standard OPV materials, but their structural ordering is complex and hence not fully understood. By applying the intensity variance analysis from fluctuation microscopy [2] to the 4D nanodiffraction data reveals the dominant peak positions raised by the ordering within the molecules, which are consistent with the expected alkyl and $\pi$ - $\pi$ stackings of P3HT (Fig 2a). Based on the identified peak positions, we performed the real space reconstruction of the nanoscale ordered regions at different $\mathrm{k}$ vectors sampling the entire reciprocal space. (Fig $2 \mathrm{~b}$ ), which provides further information on the type, size, orientation, and volume fraction of the ordering among the polymer molecules.

Figure 2c shows the 2-dimensional histogram showing the average number of the ordered domains as a function of their size and $\mathrm{k}$ value. The map shows a very detailed size distribution of the ordered domains and how they are related to the type of the ordering (which is represented by the k value). The new detailed information on molecular ordering can be directly compared to experimental data [3] to establish direct structure-property relationships. We have also applied the same method to the P3HT:PCBM blend to investigate the changes in the structure due to the addition of acceptors. For both systems, donor and donor + acceptor blend, our data indicates a general trend showing that bigger particles possess larger spacing between molecules but are slightly less frequent than smaller particles with smaller spacing between molecules. 
In summary, our new analysis provides detailed information on type, size, volume fraction, spatial and orientation distribution of the molecular ordering at the nanoscale. By providing such level of detail our technique can potentially help establish better structure-property relationships and improve the overall understanding of not just P3HT:PCBM systems but other materials that are beam sensitive and/or possess medium range ordering.
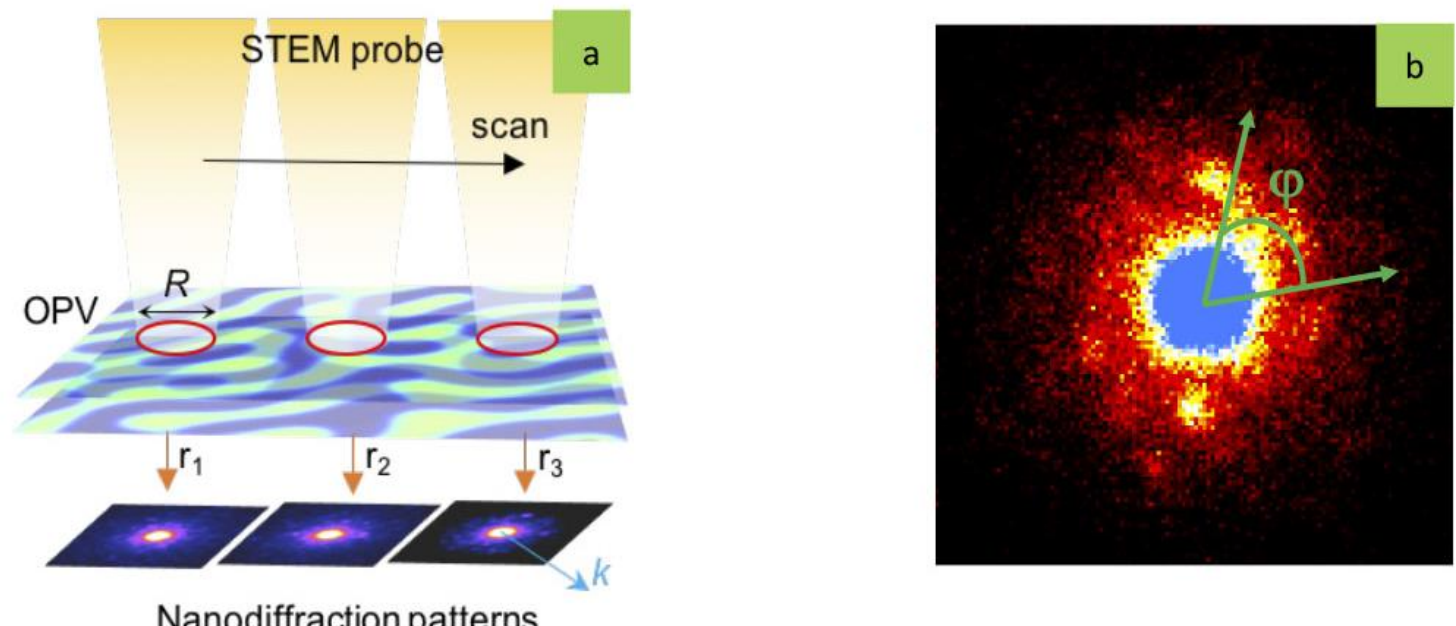

Nanodiffraction patterns

Figure 1. (a) Schematic of 4D-STEM over the polymer matrix. Different nano-diffraction patterns indicate heterogeneity of the sample. (b) Single nanodiffraction pattern from P3HT thin film.
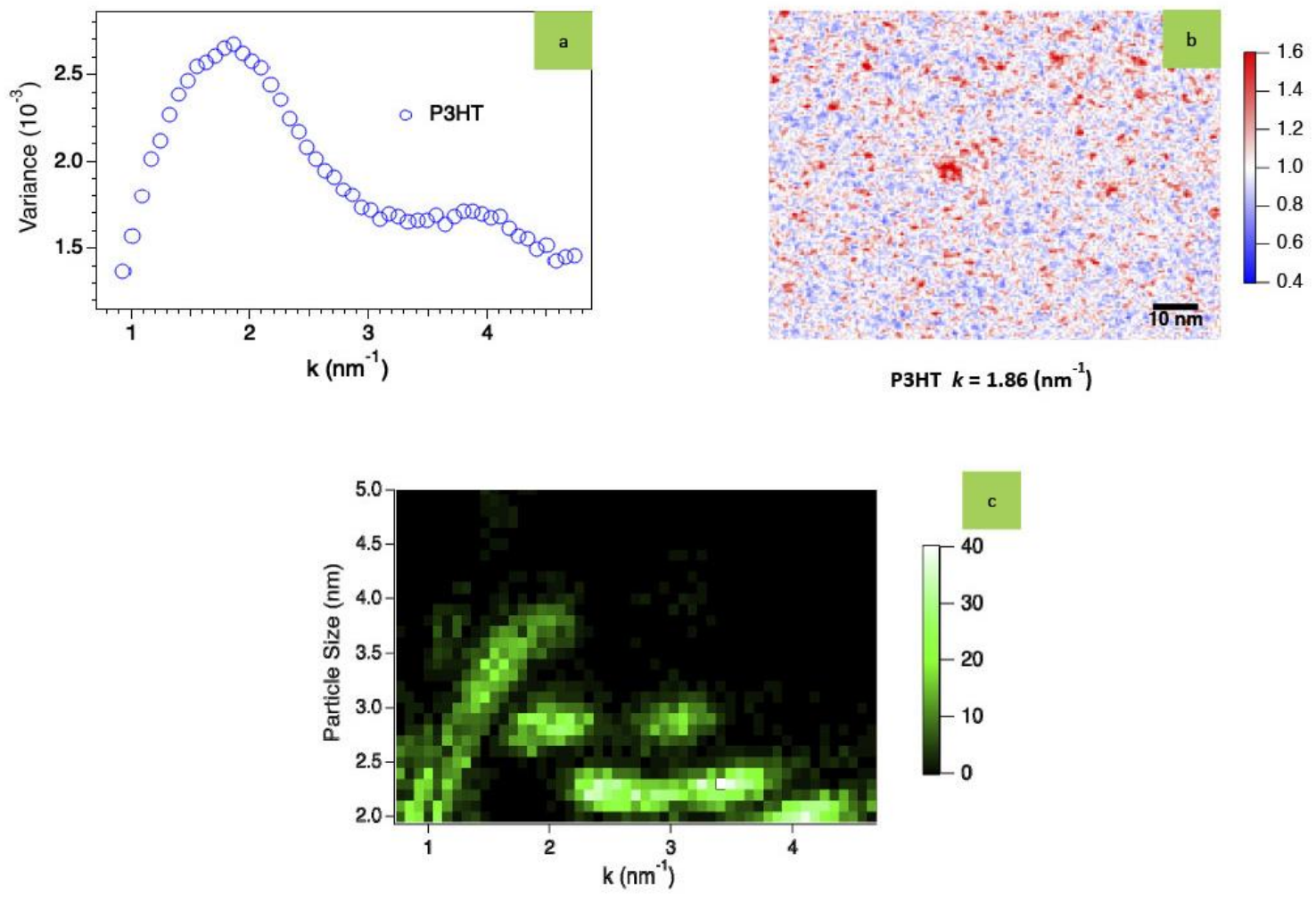

Figure 2. (a) Intensity variance of $\mathrm{P} 3 \mathrm{HT}$ after in-situ annealing at $130^{\circ} \mathrm{C}$ for $1 \mathrm{hr}$. (b) Real space reconstruction map of $\mathrm{P} 3 \mathrm{HT}$ thin film at $\mathrm{k}=1.86 \mathrm{~nm}-1$ showing diffracting domains depicted in red. (c) 
Two dimensional histogram showing agglomeration of particle sizes at different $\mathrm{k}$ space, which provides much higher level of details as compared to Fig. $2 \mathrm{a}$.

\section{References}

[1] Olivier, Y., Niedzialek, D., Lemaur, V., Pisula, W., Mullen, K., Koldemir, U., ... Beljonne, D. (2014). 25th Anniversary Article: High-Mobility Hole and Electron Transport Conjugated Polymers: How Structure Defines Function. Advanced Materials VO - 26, (14), 2119. https://doi.org/10.1002/adma.201305809

[2] Hwang, J., Melgarejo, Z. H., Kalay, Y. E., Kalay, I., Kramer, M. J., Stone, D. S., \& Voyles, P. M. (2012). Nanoscale Structure and Structural Relaxation in $\mathrm{Zr}_{50} \mathrm{Cu}_{45} \mathrm{Al}_{5}$ Bulk Metallic Glass. Phys. Rev. Lett., 108(19), 195505. https://doi.org/10.1103/PhysRevLett.108.195505

[3] Treat, N. D., Shuttle, C. G., Toney, M. F., Hawker, C. J., \& Chabinyc, M. L. (2011). In situ measurement of power conversion efficiency and molecular ordering during thermal annealing in P3HT:PCBM bulk heterojunction solar cells. J. Mater. Chem., 21(39), 15224-15231. https://doi.org/10.1039/C1JM12677F 